\title{
Évaluation de la mise en œuvre du programme d'intervention In vivo chez les jeunes de 11-12 ans d'une école primaire québécoise présentant un problème de comportements intériorisés : une étude exploratoire
}

\section{Catherine Fréchette-Simard, Jonathan Bluteau et Isabelle Plante}

Université du Québec à Montréal

\begin{abstract}
Résumé : Les troubles intériorisés représentent le problème de santé mentale le plus courant chez les jeunes de 10 à 17 ans. La présente étude exploratoire a évalué, dans une école primaire québécoise, l'implantation du programme In vivo, qui s’appuie sur les compétences à gérer le stress pour limiter les comportements intériorisés. Utilisant un devis mixte dévaluation, les participants au programme ( $n=4,11-12$ ans), leurs parents $(n=4)$ et des intervenants scolaires impliqués $(n=7)$ ont pris part à létude. Les résultats soulignent la pertinence du programme dans ce contexte et révèlent les obstacles et facilitateurs de l'implantation.
\end{abstract}

Mots clé : anxiété, évaluation de programme, école primaire, prévention, stress, troubles intériorisés

Abstract: Internalized disorders are the most current mental health issue during childhood and adolescence. This study sought to assess the implementation of In vivo, an elementary school program aiming to treat anxiety with stress management strategies. The study is based on a mixed method design and collected data from the students experiencing the program $(n=4,11-12 y .0$.), their parents $(n=4)$, and other school staff members involved $(n=7)$. The findings of the study show that the program is relevant in this specific context and also highlight the obstacles and facilitators to implementation.

Keywords: anxiety, program evaluation, elementary school, prevention, stress, internalized disorders,

\section{INTRODUCTION}

Les problèmes de comportements intériorisés, qui regroupent principalement l'anxiété et la dépression, sont très répandus chez les enfants et adolescents (Lecompte, Moss, Cyr et Pascuzzo, 2014; McLaughlin et Hatzenbuehler, 2009; Narusyte,

Correspondance à l'auteur : Catherine Fréchette-Simard, frechette-simard.catherine@ courrier.uqam.ca 
Ropponen, Alexanderson et Svedberg, 2017; Zahn-Waxler, Klimes-Dougan et Slattery, 2000). En effet, les troubles anxieux représentent le problème de santé mentale le plus courant chez cette clientèle (Albano, Chorpita et Barlow, 2003; Weissman, Antinoro et Chu, 2009), avec une prévalence pouvant atteindre jusquà $33 \%$ des jeunes au Québec et significativement plus élevée chez les filles, tous âges confondus (Piché, Cournoyer, Bergeron, Clément et Smolla, 2017). De plus, les problèmes d'anxiété sont particulièrement comorbides avec les problèmes de dépression : jusquà $70 \%$ des jeunes adolescents souffriraient à la fois de symptômes anxieux et dépressifs (Katzman et coll., 2014; Piché et coll., 2017). Lorsqu'ils ne sont pas pris en compte, les comportements intériorisés qui apparaissent durant l'enfance tendent à augmenter et à persister jusqu'à lâge adulte (Narusyte et coll., 2017), en plus d'entrainer des difficultés relatives à l'estime de soi, aux habiletés sociales (Dumas, 2012) ainsi que des conséquences scolaires négatives (Grills-Taquechel, Fletcher, Vaughn, Denton et Taylor, 2013; Wood, 2006). La période de transition primaire-secondaire représente une période critique pour ces élèves. Non seulement l'arrivée de la puberté constitue-t-elle un moment difficile pour les jeunes, en augmentant leur niveau de stress (Lupien, King, Meany et McEwen, 2001) et leur vulnérabilité à l'anxiété (McLaughlin et Hatzenbuehler, 2009), mais le passage du primaire au secondaire saccompagne de nombreux changements susceptibles d'accroître la manifestation de comportements intériorisés (ex. changement d'école, perte du cercle d'amis, attentes du milieu scolaire différentes, etc.). Par conséquent, le milieu scolaire, et plus spécifiquement la période qui précède la transition primaire-secondaire, est propice à la prévention des comportements intériorisés chez les jeunes de moins de 12 ans. Dans le cadre de la présente étude, le programme In vivo a été implanté pour la première fois dans une école primaire du Québec, auprès de jeunes de 11-12 ans présentant un problème de comportements intériorisés, et la qualité de son implantation a été évaluée. Le choix de ce programme repose notamment sur les résultats prometteurs deétudes ayant évalué son implantation auprès d'adolescents de plus de 12 ans (Bluteau, 2017; CISSS, 2016; Dufour et Bluteau, 2016a; Dufour et Bluteau, 2016b; Dufour et Bluteau, 2016c; Girolamo, 2018). Ainsi, évaluer ce programme dans un contexte scolaire primaire avec des élèves plus jeunes est primordial pour valider la faisabilité de celui-ci à lécole primaire et ainsi connaître sa pertinence auprès délèves québécois de 11-12 ans présentant une problématique de comportements intériorisés.

\section{CONTEXTE THÉORIQUE}

\section{Le programme In vivo}

Le programme In vivo, développé par le professeur et psychoéducateur Jonathan Bluteau, a vu le jour en 2012 (édité en 2015) à la suite d'un besoin des Centres Jeunesse du Québec d’améliorer les pratiques auprès des jeunes aux prises avec des problèmes intériorisés. En outre, le programme développé devait répondre aux limites des programmes actuels trop centrés sur l'acquisition des connaissances 
et peu sur la pratique de nouveaux comportements. Ainsi, le programme vise le développement de compétences pour faire face au stress et s'adresse à des jeunes de 10 à 17 ans présentant des problèmes intériorisés (anxiété, dépression) ou extériorisés (agressivité, impulsivité). Le programme comporte quatre objectifs généraux soit : 1) approfondir les connaissances sur le stress; 2) améliorer l'autorégulation en situation de stress; 3) acquérir des techniques de gestion du stress; et 4) développer l'utilisation des stratégies d'adaptation. Les dix séances du programme, à raison d'une rencontre par semaine, comprennent trois phases : l'éducation au stress, l'éducation et l'entrainement aux stratégies de coping (les stratégies d'adaptation) ainsi que l'entrainement par inoculation au stress. Le tableau 1 présente plus en détail les thématiques et le contenu abordés dans le cadre du programme.

Le programme a été qualifié d'innovant par le ministère du conseil exécutif du Gouvernement du Québec (2013), et son originalité est la combinaison de l'approche cognitivo-comportementale avec l'entrainement par inoculation au

Tableau 1. Thématiques et contenu du programme In vivo

\begin{tabular}{|c|c|}
\hline Thématique par séance & Contenu \\
\hline $\begin{array}{l}\text { 1. Brise-glace : première séance activité } \\
\text { avec le groupe }\end{array}$ & $\begin{array}{l}\text { Présentation de la structure, des } \\
\text { composantes et des objectifs du } \\
\text { programme, ainsi que des rôles et } \\
\text { responsabilités. Séance-activité réalisée } \\
\text { avec les parents }\end{array}$ \\
\hline $\begin{array}{l}\text { 2. À la découverte du stress : la } \\
\text { connaissance sur le stress }\end{array}$ & $\begin{array}{l}\text { Le stress, les connaissances sur le stress } \\
\text { ainsi que les ingrédients du stress. }\end{array}$ \\
\hline $\begin{array}{l}\text { 3. Je respire par le nez pour ma santé : } \\
\text { les impacts négatifs du stress et les } \\
\text { techniques de relaxation. }\end{array}$ & $\begin{array}{l}\text { Le stress et la mémoire, l'apprentissage, } \\
\text { la santé; la technique de respiration } \\
\text { abdominale et la cohérence cardiaque. }\end{array}$ \\
\hline $\begin{array}{l}\text { 4. On va y penser : modèle cognitif et } \\
\text { comportemental }\end{array}$ & $\begin{array}{l}\text { L'influence des évènements sur } \\
\text { les pensées, les émotions et les } \\
\text { comportements. }\end{array}$ \\
\hline $\begin{array}{l}\text { 5. Je SPIN mon stress : déconstruction } \\
\text { d'une situation stressante }\end{array}$ & $\begin{array}{l}\text { L'analyse d'une situation stressante et la } \\
\text { Grille SPIN. }\end{array}$ \\
\hline $\begin{array}{l}\text { 6. Des stratégies pour la vie : les } \\
\text { stratégies d'adaptation }\end{array}$ & $\begin{array}{l}\text { Les stratégies positives, négatives et le } \\
\text { soutien social pour gérer le stress. }\end{array}$ \\
\hline $\begin{array}{l}\text { 7. Des problèmes aux solutions : la } \\
\text { résolution de problème }\end{array}$ & $\begin{array}{l}\text { Les étapes de la résolution de problème et } \\
\text { la demande d'aide. }\end{array}$ \\
\hline $\begin{array}{l}\text { 8. Comme les cinq de la main : le } \\
\text { soutien social }\end{array}$ & $\begin{array}{l}\text { Le soutien social et le jeu de cartes: } \\
\text { Chasse aux mammouths. }\end{array}$ \\
\hline 9. Mon profil : le profil de l'élève & $\begin{array}{l}\text { Les grilles d'échelle de stress cumulées et } \\
\text { le profil de stress de l'élève. }\end{array}$ \\
\hline 10. Mes nouvelles compétences : le bilan & $\begin{array}{l}\text { Les réussites et les nouvelles } \\
\text { compétences, séance-activité réalisée } \\
\text { avec les parents. }\end{array}$ \\
\hline
\end{tabular}


stress, lequel consiste à entrainer le jeune en situation de stress réel et contrôlé. En effet, le programme se démarque de la grande majorité des interventions offertes, lesquelles se fondent généralement de façon exclusive sur un enseignement théorique (Barrett et Ollendick, 2004, Fréchette-Simard, Plante et Bluteau, 2018). In vivo recourt à l'entrainement par inoculation au stress, à l'aide de modules d'entrainement. Ces modules représentent des activités à prise de risque comme un trampoline, un mur d'escalade, une piscine avec tremplin, etc. Le recours aux modules permet de proposer divers défis aux participants afin de déclencher quatre déterminants du stress : le sentiment de contrôle diminué, la perception de la personnalité menacée, l'imprévisibilité et la nouveauté (Dickerson et Kemeny, 2004; Mason, 1968). La démarche proposée consiste en un véritable entrainement actif à l'autorégulation, ce qui favoriserait davantage le développement des compétences adaptatives (Barlow, Allen et Choate, 2004; Barlow et coll., 2011). Pour pouvoir dispenser le programme, les animateurs doivent, au préalable, avoir suivi une formation théorique et pratique d'une durée de deux jours. De plus, un manuel détaillé, mis à la disposition des animateurs, inclut les objectifs de chaque séance-activité, le matériel nécessaire, le temps dédié à chaque étape du programme, de même que des consignes pour les animateurs, telles que les attitudes et façons de faire à adopter.

À la suite de son implantation, plus de 200 jeunes ont suivi le programme dans divers contextes, notamment dans deux Centres Jeunesse situés au Québec, un Institut thérapeutique éducatif et pédagogique en France et une école secondaire québécoise, le programme In vivo a répondu aux critères d'un programme prometteur auprès des adolescents de 12 à 17 ans, présentant un trouble intériorisé (Bluteau, 2017; CISSS, 2016; Dufour et Bluteau, 2016a; Dufour et Bluteau, 2016b; Dufour et Bluteau, 2016c; Girolamo, 2018). Par exemple, Bluteau (2017) rapporte une diminution de l'utilisation des stratégies négatives, de même qu'une diminution significative des symptômes intériorisés chez les garçons. Cependant, le programme na pas encore été évalué chez une population délèves de moins de 12 ans, ni durant la période de transition entre le primaire et le secondaire. Aussi reste-t-il à évaluer la faisabilité et la qualité de sa mise en œuvre dans un contexte scolaire primaire.

\section{Théorie du programme de Chen}

Dans une optique de continuité avec les études évaluatives ayant précédemment évalué le programme In vivo, la théorie du programme de Chen (2005) a été ciblée comme cadre de référence pour lévaluation de la mise en œuvre de ce programme dans la présente étude. Cette théorie est fréquemment utilisée pour lévaluation de programme puisqu'elle permet de produire de nouvelles connaissances et de mieux comprendre les mécanismes d'action du programme et son processus de changement (Chen, 2005). En effet, ce cadre, schématisé dans la figure 1 , permet dévaluer de façon systématique les différentes variables qui contribueront à l'efficacité d'un programme et de valider la théorie du programme. Chen (2005) évalue, en deux phases distinctes, d'une part la fidélité de l'implantation 


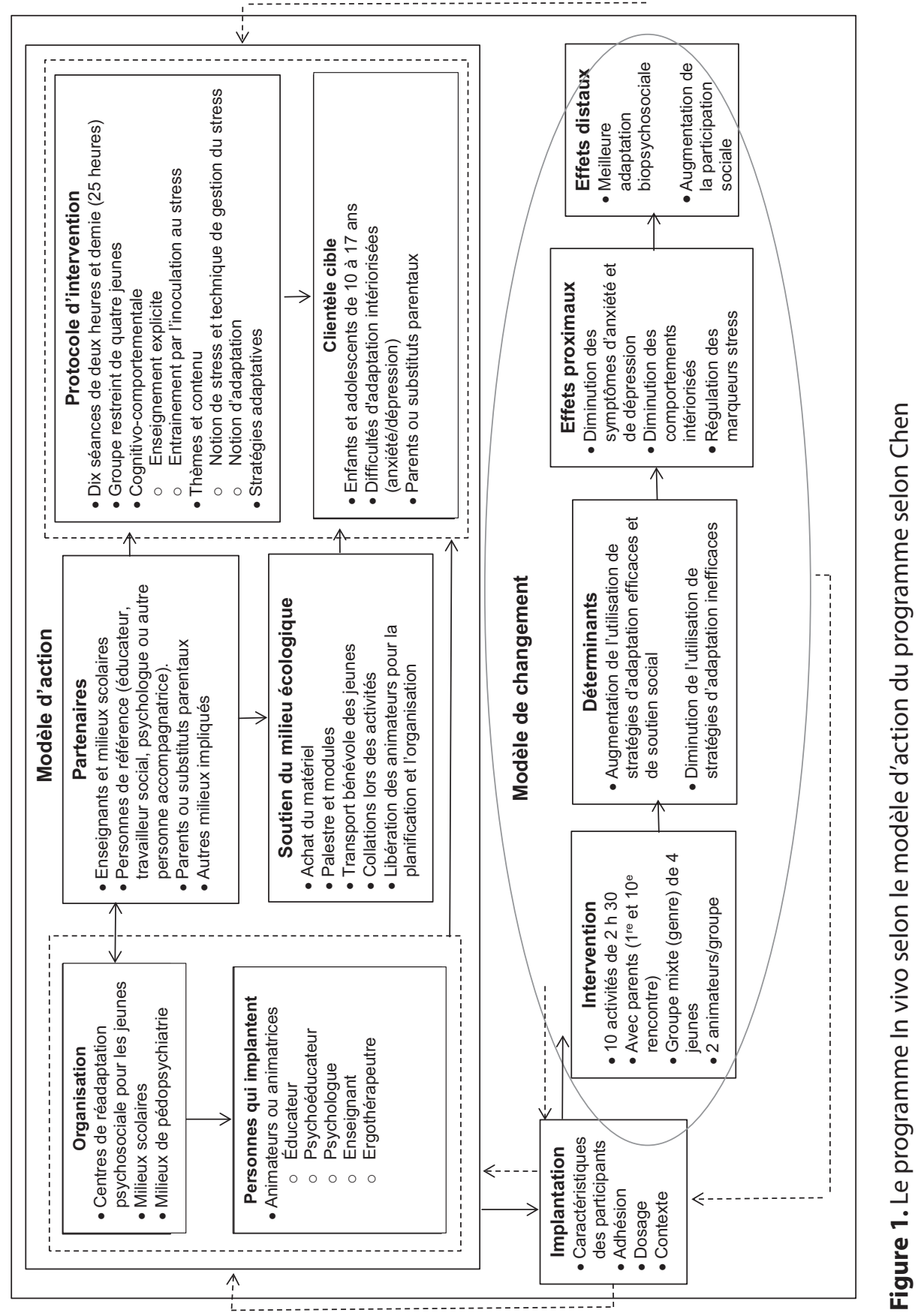


et la faisabilité du modèle d'action du programme et, d'autre part, les effets sur les participants, ce qu'il appelle le modèle de changement. Dans le cadre de la présente étude, seul le modèle d'action a été utilisé, de façon à évaluer chacune des composantes qui contribuent à l'efficacité du programme. Ces composantes sont logiquement liées les unes aux autres et chacune d'entre elles contribue au succès de l'implantation. La figure 1 présente le programme In vivo selon le modèle d'action du programme selon Chen (2005), plus précisément il a servi de cadre d'analyse pour la présente évaluation. On y retrouve également le modèle de changement, lequel n’a pas été évalué dans cette étude.

\section{OBJECTIFS DE L'ÉTUDE}

Dans un contexte de validation de pratique visant un processus d'amélioration continue du programme, l'objectif général de cette étude consistait à évaluer, dans une visée exploratoire, la mise en œuvre du programme In vivo dans une école primaire québécoise, chez des élèves de $6^{\mathrm{e}}$ année de 11-12 ans présentant une problématique de comportements intériorisés. Plus précisément, l’étude comportait deux objectifs spécifiques : 1) évaluer la fidélité de l'implantation (l'adhésion des animatrices et des participants au programme, le degré d'exposition, la qualité de l'intervention) et 2) évaluer la mise en œuvre du programme à travers les six composantes du modèle d'action de Chen (2005, voir figure 1). Considérant l'absence de données relatives à l'implantation de ce programme au primaire, la présente étude viendra combler un manque évident sur la connaissance du programme et son modèle d'action. De plus, les résultats pourront orienter l'intervention en milieu scolaire primaire afin de limiter les problèmes de comportement intériorisés chez les jeunes de 11-12 ans.

\section{MÉTHODOLOGIE}

Afin de répondre aux objectifs fixés, un devis mixte d'évaluation de programme avec échantillon de convenance a été utilisé. Létude a été réalisée en collaboration avec une commission scolaire et un certificat éthique du comité d'éthique à la recherche de l'Université du Québec à Montréal a été obtenu pour sa réalisation.

\section{Participants}

Léchantillon disponible a été limité par certaines contraintes liées au programme ainsi qu'au milieu. En effet, le programme prévoit un ratio de deux animateurs pour quatre participants, alors que le milieu scolaire d'accueil ne disposait pas des ressources pour offrir le programme à plus de quatre participants. Létude incluait toutefois d'autres acteurs qui ont participé à l'évaluation de la mise en œuvre du programme.

Ainsi, les participants à l'étude, recrutés dans une école de milieu urbain favorisé, étaient 1) quatre élèves (deux filles et deux garçons) de $6^{\mathrm{e}}$ année du primaire $(M=11,5$ ans, $E T=1,0)$ qui présentaient un problème de comportements 
intériorisés, 2) les parents de ces élèves ( $n=4$, trois femmes et un homme), 3 ) les animatrices du programme $(n=2), 4)$ les enseignants de ces élèves $(n=3$, une femme et deux hommes) et 5) la direction de l'école ( $n=2$, deux femmes).

Les participants élèves de l'étude ont été recrutés afin de correspondre aux deux critères d'inclusion de l'étude, à savoir l'âge et la présence d'un profil de comportements intériorisés. Ainsi, les élèves ont été sélectionnés parmi une population de 75 élèves de $6^{\mathrm{e}}$ année, âgés de 11-12 ans. Dix élèves ont d'abord été ciblés par la psychoéducatrice de l'école, en raison de leurs manifestations de comportements intériorisés. Dans le cadre de leur suivi individuel avec leur psychoéducatrice, ces derniers ont été évalués à l'aide des sous-échelles de comportements intériorisés du questionnaire Child Behavior Checklist (CBCL, Achenbach et Rescorla, 2001). Afin de correspondre au ratio prévu du programme, les quatre participants ayant obtenu le plus haut score au CBCL ont été proposés pour l'étude. Plus spécifiquement, selon les seuils de l'outil, un des élèves retenus atteignait le seuil clinique, deux d'entre eux avaient un score jugé à risque et le dernier avait un seuil sousclinique. À noter également que l'étude comportait les critères d'exclusion suivants : l'inclusion dans une autre intervention visant le stress ou l'anxiété, la manifestation d'un problème de santé physique ou mentale (autre que l'anxiété ou la dépression) ou un retard cognitif qui aurait limité la participation au programme. Ces critères d'exclusion, déterminés par le programme, sont liés au fait que les activités d'inoculation au stress ont été jugées trop risquées pour des élèves présentant ces profils. Dans la présente étude, aucun élève n’a été exclu sur la base de ces critères.

Les participants adultes incluaient les deux animatrices, soit une psychoéducatrice (A1) et une enseignante (A2), qui ont suivi la formation préalable permettant de dispenser le programme. Les autres participants adultes (parents, enseignants, direction) ont joué un rôle secondaire dans l'étude, soit dans la documentation de l'appréciation et des effets perçus du programme. Conformément au protocole du programme, un seul parent par élève était présent.

\section{Procédure d'implantation du programme et collecte de données}

Le processus de recherche s'est décliné en deux procédures distinctes, soit l'implantation du programme ainsi que la collecte de données. L'intervention a eu lieu sur une période de dix semaines à raison d'une séance de $2 \mathrm{~h} 30$ par semaine entre $14 \mathrm{~h} 30$ à $17 \mathrm{~h}$. Ces séances se sont déroulées chacune en deux phases, la première en salle de classe et la seconde en gymnase dans un centre d'escalade situé à proximité de l'école. Le choix de se tourner vers des activités d'escalade a été fait en raison de l'accessibilité du centre qui se trouvait à distance de marche de l'école. La procédure de collecte de données s'est déroulée en trois phases : 1) avant l'intervention (questionnaires sociodémographique et $\mathrm{CBCL}$ ), 2) durant l'intervention (questionnaires d'autoévaluation de l'implantation et journal de bord des animatrices, à la fin de chaque séance) et 3) après l'intervention (questionnaires d'autoévaluation de la fidélité et du modèle d'action, questionnaires d'adhésion des participants, CBCL et entrevues). Le détail des différentes mesures utilisées est décrit dans la prochaine section. 


\section{Mesures}

Un questionnaire sociodémographique a été rempli par les élèves et leur parent avant le début du programme pour recueillir des données concernant le genre du participant, son âge, son niveau scolaire et son état de santé physique et mentale.

\section{Problèmes de comportements intériorisés}

Le questionnaire CBCL a été utilisé pour déterminer le profil intériorisé des participants. Pour ce faire, les trois échelles mesurant les comportements intériorisés $(\alpha=0,91)$ ont été retenues, soit l'échelle d'anxiété/dépression, l'échelle de retrait social/dépression et l'échelle de plaintes somatiques. Pour chacun des 32 items de ce questionnaire, l'enfant répond à l'aide d'une échelle de Likert à trois points, allant de « 1 = ne s'applique pas », à « 3 = s'applique toujours ou souvent ». Les participants élèves ont eux-mêmes rempli le questionnaire, sous la supervision de la psychoéducatrice.

\section{Fidélité d'implantation du programme}

À la fin de chacune des séances du programme, les animatrices ont répondu à un questionnaire maison d'autoévaluation de l'implantation incluant trois questions ayant pour but de recenser les commentaires concernant 1) les objectifs des séances, 2) la participation des jeunes ainsi que 3) le degré de conformité du contenu par rapport à ce qui est prévu au programme. Dans l'optique de documenter la fidélité de chacune des étapes de l'implantation, les animatrices ont également consigné leurs observations dans un journal de bord.

À la fin du programme, un questionnaire autorapporté évaluant la fidélité de l'implantation (Joly, 2008; Paquette, Joly et Tourigny, 2009) a été rempli par les animatrices. Le questionnaire se divise en deux parties sur la fidélité de l'implantation. La première partie comporte cinq sections : 1) l'adhésion des animateurs, 2) le degré d'exposition (dosage), 3) la qualité de l'intervention, 4) le degré de participation et 5) l'adaptation du programme. La seconde partie se divise en quatre sections, soit le programme, l'organisation, les animatrices ainsi que les participants. Ces deux parties comportent chacun des items auxquels les animatrices doivent répondre à l'aide d'une échelle de Likert en 10 points, allant de « 0 = pas du tout » à " $10=$ tout à fait ». Un score de $75 \%$ et plus obtenu aux différents indicateurs d'adhésion, de dosage et de participation correspond à un résultat jugé très fidèle à ce qui était prévu au programme. Un score se situant entre $61 \%$ et $74 \%$ est jugé moyennement fidèle alors qu'un score de $60 \%$ et moins est considéré comme étant peu fidèle (Paquette et coll., 2009). Le questionnaire a procuré des coefficients alpha supérieurs à 0,80 pour toutes les échelles (voir Gamache, Joly et Dionne, 2011).

Dans le but de documenter l'adhésion des participants au programme, les participants élèves ont répondu à une adaptation du questionnaire autorapporté du Child's Evaluation Inventory (CEI) de Kazdin, Siegel et Bass (1992), afin de recueillir de l'information sur leur adhésion au programme et ainsi de connaître la perception de leur expérience. Le score total de l'échelle d'adhésion $(\alpha=0,89)$ 
est obtenu à l'aide de deux sous-échelles, soit la Perception des progrès réalisés $(\alpha=0,95)$ et l'Acceptabilité $(\alpha=0,95)$ (Bluteau, 2017). Le questionnaire comporte 19 items fermés, qui comportent des échelles de Likert variées, suivis d'une question à court développement permettant de recueillir des commentaires.

\section{Modèle d'action du programme}

Le questionnaire d'autoévaluation du modèle d'action du programme a été utilisé auprès des animatrices (Gamache et coll., 2011). Il comporte six sections documentant : 1) l'implantation auprès d'élèves recevant l'intervention, 2) le processus, 3) les personnes qui implantent le programme, 4) lorganisation, 5) les partenaires ainsi que 6) le contexte écologique. Le questionnaire comporte des questions ouvertes ainsi que des questions à choix de réponse avec des échelles de Likert en 10 points, allant de « $0=$ pas du tout » à « $10=$ tout à fait ». Ce questionnaire, élaboré et utilisé par Gamache est ses collègues (2011) pour évaluer la fidélité d'implantation d'un programme implanté au Québec, n’a pas encore fait l'objet d'une validation empirique, mais sa validité de construit s'appuie directement sur le choix des questions qui couvrent les six composantes du modèle de Chen (2005).

Des entrevues semi-dirigées ont été réalisées avec les participants élèves afin de recueillir des données sur leur expérience. L’entrevue portait sur le programme en général, sur les stratégies d'intervention, sur les changements perçus, sur leur implication personnelle, sur les modules d'escalade ainsi que sur le stress généré par l'escalade.

Les participants adultes de l'étude ont répondu à des questions d'appréciation du programme, lors d'entrevues semi-dirigées, afin de documenter leur appréciation de l'intervention ainsi que les effets perçus quant à l'amélioration des comportements des élèves.

\section{Analyses}

Pour répondre au premier objectif de l'étude, des scores moyens et écarts-types ont été extraits et calculés à l’aide du logiciel Excel, à partir des données issues des questionnaires de fidélité de l'implantation et des questionnaires d'adhésion des participants. Ensuite, en réponse au second objectif de recherche, chaque composante de l'implantation a fait l'objet d'une évaluation qualitative plus spécifique suivant le modèle de Chen (2005), sous la forme d'une analyse de contenu thématique, à l'aide du logiciel NVivo. Pour ce faire, le contenu des entrevues semi-dirigées a d'abord été retranscrit en verbatim. Ces derniers, de même que les données qualitatives issues des différents questionnaires d'autoévaluation de l'implantation, d'autoévaluation du modèle d'action et du journal de bord ont ensuite été consignées.

\section{RÉSULTATS}

Les résultats sont présentés dans les deux prochaines sections, suivant la présentation des objectifs de l'étude, soit l'évaluation 1) de la fidélité de l'implantation et 
2) du modèle d'action. Les participants sont ci-après désignés par les abréviations $\mathrm{P} 1, \mathrm{P} 2, \mathrm{P} 3$ et $\mathrm{P} 4$.

\section{Fidélité de l'implantation}

Les résultats sont présentés en quatre sections : 1) l'adhésion des animatrices, 2) l'adhésion des participants, 3) le degré d'exposition et 4) la qualité d'intervention. Les scores moyens des différentes sections présentées permettront de qualifier la fidélité de l'implantation en fonction des seuils de Paquette et coll. (2009).

\section{Adhésion des animatrices}

Le tableau 2 présente les statistiques descriptives provenant des scores des deux animatrices concernant leur adhésion au programme. Ces résultats montrent, avec un score moyen de $80,8 \%$, que dans l'ensemble, le cadre théorique du programme a été respecté, de même que les contenus abordés, les activités présentées et l'instrument d'évaluation proposé.

\section{Adhésion des participants}

Le score d'adhésion au questionnaire CEI s'est avéré élevé pour P1 (89/95), mais plus bas pour les trois autres participants $(\mathrm{P} 2=54 / 95 ; \mathrm{P} 3=73 / 95 ; \mathrm{P} 4=60 / 95)$. En entrevue, tous ont rapporté avoir fait des acquis à la suite du programme et trois des quatre participants ont rapporté avoir préféré l'entrainement en gymnase aux activités structurées. De plus, le score de 6,5/10 pour l'item relatif à l'obtention de la participation attendue révèle, selon les animatrices, que la participation des élèves nétait pas optimale.

\section{Degré d'exposition}

Les résultats présentant le degré d'exposition se trouvent dans le tableau 3, qui rapporte un score moyen de $71 \%$. On constate que la durée des activités a dû être modifiée par rapport à ce qui était prévu (score de 3,5/10). En effet, les données qualitatives ont montré que plusieurs adaptations ont été nécessaires afin de

Tableau 2. Résultats des questions concernant l'adhésion des animatrices

\section{Questions}

Moyenne/10 (ÉT)

Les étapes de réalisation des activités ont été suivies.

$8,0(0)$

Les principes théoriques en lien avec les activités à réaliser et

le changement attendu ont été respectés dans les activités.

Les contenus spécifiques à aborder ont été respectés.

$9,0(1,4)$

Les consignes à suivre lors des activités ont été respectées.

Les activités prescrites par le programme ont été effectuées.

Les outils d'évaluations proposés par le programme ont été utilisés.

TOTAL (sur 60)

Pourcentage de la moyenne 
Tableau 3. Résultats des questions concernant le degré d'exposition au programme

\section{Questions}

La durée prévue des activités a été respectée.

La fréquence prévue des activités a été respectée.

Les différents types d'activités ont été réalisés.

La collaboration avec les partenaires a été faite comme prévu.

L'intervention a été faite comme prévu auprès des différents

types de participants (élèves et parents).

TOTAL (sur 50)

Pourcentage de la moyenne
Moyenne/10 (ÉT)

$3,5(0,7)$

$9,0(1,4)$

$8,0(2,3)$

$7,5(3,5)$

$7,5(0,7)$

$35,5(2,1)$

$71 \%$

Tableau 4. Résultats des questions concernant la qualité de l'intervention

\section{Questions}

Les attitudes particulières identifiées par le programme ont été adoptées.

Les manières de faire identifier par le programme ont été respectées.

TOTAL (sur 20)

Pourcentage de la moyenne
Moyenne /10 (ÉT)

$8,0(0)$

$8,0(0)$

$16(0)$

$80 \%$

s'ajuster aux besoins des participants (ex. : plus de temps pour répondre aux questions posées lors des activités, plus de temps pour s'habiller en hiver) de même qu’aux imprévus relatifs à l'horaire scolaire (ex. : examen à terminer, évènement spécial en classe, etc.).

\section{Qualité de l'intervention}

Dans le questionnaire dévaluation de la fidélité, les animatrices rapportent, avec un score moyen de $80 \%$, que les attitudes à adopter et les façons de faire prescrites par le programme ont dans l'ensemble été respectées, comme rapporté dans le tableau 4. De plus, en entrevue et dans le journal de bord, les animatrices ont mentionné avoir vécu des difficultés liées à la nouveauté du programme dans leur pratique, à la conciliation des particularités de chaque participant, de même quà la gestion des problèmes de discipline.

\section{Modèle d'action}

\section{Organisation}

Les entretiens réalisés auprès de la direction d'école ont révélé un portrait positif de l'implantation du programme. Toutefois, à la question « La direction a fourni les ressources matérielles suffisantes? » de la section Organisation du 
Tableau 5. Résultats des questions concernant la dimension Intervenants

J'ai des appréhensions face au programme.

Je perçois le programme de manière positive.

Grâce à ce programme, je vais enrichir mon travail.

$10,0(0)$

Ce programme va m'apporter des bénéfices personnels.

$10,0(0)$

Ce programme aura des effets positifs sur les participants.

$8,5(2,1)$

Je crois qu'il est possible d'appliquer un tel programme dans

$4,5(2,1)$

mon milieu.

questionnaire de fidélité de l'implantation, un score moyen de 6/10 $(E ́ T=5,7)$ a été obtenu. Les données du journal de bord des animatrices ont précisé que l'engagement de la direction dans l'implantation a été faible et que peu de supervision a été offerte pour soutenir les intervenantes dans ce processus, notamment parce que le matériel pédagogique nécessaire a été fourni par la présente recherche.

\section{Personnes qui implantent}

Le tableau 5 présente les résultats à la dimension Intervenants du questionnaire de fidélité de l'implantation. En dépit de scores plutôt élevés, la croyance qu'il est possible d'appliquer un tel programme dans le milieu est plutôt faible, avec une moyenne de 4,5/10 (ÉT = 2,1). En entrevue, des difficultés de gestion du temps ont été soulevées par rapport à la tâche planifiée.

\section{Partenaires}

La collaboration avec les partenaires a été évaluée à l'aide du questionnaire de fidélité de l'implantation et suggère que celle-ci sest avérée plutôt positive. Cependant, les animatrices ont toutes deux mentionné, en entrevue et dans le journal de bord, qu'il serait souhaitable de mieux expliquer le programme aux enseignants et de préciser le rôle de chacun des intervenants, afin d’optimiser leur participation en réinvestissant en classe les stratégies apprises.

\section{Contexte écologique}

Le journal de bord des animatrices a permis de montrer que dans l'ensemble, le contexte écologique a été favorable à l'implantation du programme, notamment grâce à un local pour la partie théorique ainsi que des collations fournis gratuitement par l'école de même qu’à l'accès gratuit à un centre d'escalade, situé à distance de marche de lécole. Paradoxalement, le fait de devoir quitter l'école a été perçu comme une contrainte importante, étant donné le temps à prévoir pour s'habiller en fonction de la température hivernale. Également, lenvironnement du centre d'escalade était peu propice à l'intervention compte tenu de la présence d’autres clients au centre et de l'aménagement physique des lieux : « Nous manquons 
d'espace et ce n'est pas l'endroit idéal pour avoir le cartable en main et écrire » (Journal de bord_A2).

\section{Protocole d'intervention}

En ce qui a trait à la perception des animatrices quant à la fidélité de l'implantation, les scores $(M=9,0 / 10, E ́ T=1,4)$ montrent que selon elles, le programme a globalement été implanté comme prévu. Toutefois, les données provenant des questionnaires d'autoévaluation de l'implantation, de même que des entrevues et du journal de bord des animatrices relèvent que plusieurs adaptations ont été faites afin d'adapter le langage à l'âge des participants et d'ajouter du temps supplémentaire pour l'intégration des techniques cognitivo-comportementales. En dépit des adaptations rapportées, les propos des animatrices (entrevues et journal de bord) suggèrent que les interventions prévues dans le protocole du programme étaient adéquates pour la clientèle ciblée: «Je sens toutefois que la partie théorique intéresse bien les élèves et convient quand même bien à leur niveau » (Journal de bord_A2).

Dans un autre ordre d'idées, plusieurs éléments du protocole d'intervention se sont révélés être facilitants, notamment l'activité d’escalade, qui a favorisé la motivation et l'adhésion des participants, ceux-ci ayant nommé l'escalade comme étant l'élément qu'ils préféraient dans le programme. P1 a rapporté en entrevue que les activités de gestion du stress sur les modules d'escalade étaient utiles au transfert des connaissances dans son quotidien. Le journal de bord des animatrices et les questionnaires d'autoévaluation de l'implantation révèlent que l'entrainement a été particulièrement utile pour pratiquer les stratégies d'adaptation enseignées (ex. " travailler fort pour réussir », " demander de l'aide », " avoir une pensée positive », etc.), et moins pour pratiquer les stratégies de gestion du stress (ex. techniques de respiration, de déconstruction du stress, etc.). Contrairement aux propos des animatrices, les entrevues avec les élèves participants et avec leurs parents indiquent plutôt que les stratégies les plus réinvesties par les élèves à la maison sont les techniques de respiration et les stratégies d'adaptation mentionnées précédemment.

Les données du journal de bord soulignent aussi l'impact positif des CFM, par l'aspect attractif pour les jeunes et le soutien à la prise de conscience des réactions de stress sur leur rythme cardiaque. Toutefois, puisque les CFM étaient destinés à des adultes, la lecture de la fréquence cardiaque procurait des résultats instables, nécessitant ainsi plusieurs ajustements pour convenir à la petite cage thoracique des participants.

\section{Clientèle visée}

La procédure de sélection, sur la base du questionnaire CBCL, a permis de recruter des jeunes qui présentaient un profil intériorisé, ce que les données du journal de bord ont également permis de corroborer. En effet, P1 présentait principalement un problème au plan de l'anxiété de performance de même qu'un stress quotidien élevé, ce qui avait tendance à augmenter son anxiété. P3 manifestait un 
niveau de stress et d'anxiété se traduisant principalement par de la passivité et de l'agitation. Il en était de même pour P4 qui démontrait un niveau de passivité particulièrement élevé durant les séances du programme, en plus de présenter d'autres problématiques non diagnostiquées qui entravaient ses relations avec les adultes et qui se traduisaient par des problèmes de comportement à l'école. P2 manifestait également de l'anxiété et du stress, caractérisés principalement par de l'évitement et un degré de passivité accru face aux difficultés vécues. Les animatrices ont rapporté certains défis liés aux caractéristiques des élèves : «Les enfants choisis ont eu de la difficulté à identifier et verbaliser les situations de stress qu'ils vivaient. " (Questionnaire modèle d'action_A1), "Il a été difficile d'obtenir un niveau d'introspection suffisant de leur part " (Questionnaire modèle d'action_A2). Elles ont également rapporté qu'il y avait beaucoup de discipline à faire et que leur participation aux activités en classe était limitée, principalement à cause de leurs difficultés à s'exprimer. Finalement, la participation des parents, prévue à la première et la dernière séance, a été faible, compte tenu notamment de leur horaire de travail incompatible avec les rencontres prévues et du manque de stationnement à proximité du centre d'escalade.

\section{DISCUSSION}

Dans l'ensemble, cette évaluation de programme a permis de documenter la faisabilité du programme In vivo pour la clientèle visée, tout en faisant ressortir des pistes d'adaptation à prévoir pour des utilisations ultérieures et la pertinence de poursuivre les études d'implantation dans ce même contexte.

\section{Fidélité de l'implantation}

Les résultats montrent que le programme a été implanté de façon relativement fidèle. En effet, les scores obtenus pour l'adhésion des animatrices, et la qualité de l'intervention ont été évalués comme étant très fidèles, avec des scores supérieurs à $75 \%$ (Paquette et coll., 2009). Le score concernant le degré d'exposition (71\%) a été évalué comme moyennement fidèle, puisqu’il se situe entre 61 et $74 \%$.

Par ailleurs, plusieurs raisons peuvent expliquer que le degré d'exposition ait été jugé moyen, tels le temps perdu durant les périodes de transition, l'importance accrue accordée à la théorie plutôt qu'à la pratique ainsi que la faible participation des élèves, des résultats cohérents avec les études antérieures réalisées avec le programme In vivo (Bluteau, 2017; Girolamo, 2018). En dépit des changements apportés quant à la durée destinée aux activités, pratiquement toutes les activités prévues ont été réalisées, soulignant ainsi la faisabilité du programme dans les délais prévus.

Concernant l'adhésion au programme, des écarts importants ont été observés selon les participants, suggérant que les profils initiaux de comportements intériorisés, eux-mêmes plutôt variés, pourraient influencer leur degré d’adhésion. En effet, le participant qui a le plus adhéré au programme semblait présenter des capacités d'introspection et une maturité accrues. Il y aurait donc lieu de vérifier, 
auprès d'un plus grand échantillon, si lâge et la maturité des participants ont une réelle influence sur l'implantation et les effets du programme. Par ailleurs, pour les élèves qui détiennent un profil intériorisé davantage inhibé, certaines recommandations peuvent être émises, notamment de leur donner davantage doutils pour faciliter leur réflexion face aux sujets abordés.

\section{Organisation, animatrices, partenaires et contexte écologique}

Puisque la mise en œuvre du programme s'insérait dans une recherche et que plusieurs ressources matérielles ont été fournies gratuitement, elle n'a pas été réalisée en contexte naturel. Le présent projet ne permet donc pas de confirmer que le milieu possédait les ressources pour mener à terme le programme, ce qui représente pourtant un aspect essentiel d'une implantation réussie (Joly, Tourigny et Thibodeau, 2005). En dépit de ce contexte de mise en œuvre particulier, plusieurs éléments soulevés par Joly et ses collègues (2005) comme étant favorables à une implantation réussie ont été documentés dans la présente étude : louverture et le soutien du milieu scolaire, lattitude positive et la motivation des animatrices. Toutefois, comme il s'agissait d'une première expérience de mise en œuvre dans cette école et pour cette clientèle, il aurait été bénéfique d’avoir un comité de pilotage afin de soutenir la démarche, comme recommandé dans la formation au programme. Également, de courtes rencontres de suivi ou des communications régulières auraient pu être incluses afin d'informer les enseignants des stratégies vues dans le cadre du programme et ainsi de favoriser le transfert des connaissances dans la classe.

\section{Protocole d'intervention}

Les différents résultats présentés précédemment montrent que deux des quatre objectifs généraux du programme semblent avoir été atteints, soit lobjectif d'améliorer les connaissances sur le stress et celui d’améliorer l'utilisation des stratégies d'adaptation. En effet, chacun des quatre jeunes a amélioré ses connaissances sur le stress et l'utilisation de certaines stratégies positives d'adaptation en situation de stress. Malgré le fait que certaines adaptations aient été nécessaires pour bien répondre aux caractéristiques des participants, le protocole semble donc être faisable pour la clientèle ciblée.

Par ailleurs, le protocole d'intervention prévoyait l'utilisation d'une stratégie innovante : l'entrainement par inoculation au stress. Or, la mise en œuvre de cette stratégie a été complexifiée par la difficulté des animatrices à proposer des défis générant suffisamment de stress aux participants lors des activités descalade. En effet, selon les formateurs au programme, les expériences d'implantation montrent qu'il faut une expérience d'animation auprès de trois à quatre cohortes avant dêtre suffisamment à l'aise avec le matériel (modules et CFM), ainsi que les stratégies d’animation (Bluteau, 2017; CISSS, 2016; Girolamo, 2018). Alors que le programme prévoyait une utilisation accrue des stratégies de gestion du stress (ex. respiration, déconstruction du stress, etc.) sur les modules d'entrainement, ces derniers ont servi davantage à mettre en pratique les stratégies d'adaptation 
pour résoudre un problème plutôt que pour gérer le stress. En particulier, la technique de respiration a été difficile à utiliser, possiblement parce que les élèves étaient très intéressés par l'escalade et ainsi moins enclins à prendre des pauses pour réfléchir et mettre en pratique les stratégies enseignées. Ainsi, les mises en œuvre ultérieures devraient prévoir davantage de pauses destinées au processus de réflexion des jeunes, pour ainsi s'assurer d'une acquisition plus optimale des stratégies proposées. De plus, augmenter la complexité des tâches graduellement durant les séances serait souhaitable pour accroître le stress perçu par les jeunes et ainsi favoriser l'inoculation au stress, au cœur du programme In vivo.

Les résultats présentés concernant les CFM vont dans le même sens qu'une précédente étude (Bluteau, 2017) qui concluait que les CFM étaient attrayants et utiles. Compte tenu des difficultés d'utilisation rencontrées compte tenu de la petite taille des participants de la présente étude, il serait pertinent de prévoir des CFM mieux adaptés, de grandeur enfant, à ce groupe d'âge.

\section{Clientèle visée}

Le fait que la clientèle visée présente des caractéristiques propres à un profil comportemental intériorisé, notamment de la timidité et de l'inhibition, pourrait expliquer certaines des difficultés rencontrées. Ainsi, il est possible que les difficultés vécues par les participants durant le programme aient été liées à leurs caractéristiques personnelles plutôt qu'à leur jeune âge. En ce sens, le programme pourrait potentiellement convenir à une clientèle de $6^{\mathrm{e}}$ année présentant des comportements intériorisés, moyennant certains ajustements en fonction de leurs capacités d'introspection ou besoins spécifiques.

\section{CONCLUSION}

Dans le cadre cette étude exploratoire, le programme In vivo a été testé et implanté pour la toute première fois dans un contexte d'école primaire québécoise, auprès délèves de $6^{\mathrm{e}}$ année qui présentent une problématique de comportements intériorisés. L'étude exploratoire évaluant sa mise en œuvre a permis de mieux comprendre les déterminants reliés à la faisabilité d'implantation dans ce contexte. Somme toute, les résultats ont révélé que la fidélité de l'implantation s'est avérée fidèle à ce qui était prévu au programme et que l'évaluation de sa mise en œuvre, à l'aide du modèle de Chen, semble réalisable en contexte primaire, avec la clientèle visée.

En dépit de ces résultats positifs, la présente étude comporte certaines limites qu'il convient de reconnaître et qui pourront orienter la recherche future. Une première limite de cette évaluation concerne la petite taille d'échantillon, qui découle principalement des contraintes du programme In vivo, destiné à quatre participants. D’autres évaluations incluant de plus grands échantillons seront donc nécessaires, pour évaluer la généralisabilité des résultats auprès de clientèles diversifiées pour ainsi confirmer la validité du programme In vivo en contexte primaire. De plus, certaines adaptations seraient souhaitables afin de favoriser l'atteinte des objectifs ciblés par le programme, tels des ajustements pour mieux tenir 
compte des besoins des élèves inhibés, une meilleure organisation des contenus théoriques et pratiques et des mesures additionnelles pour favoriser l'implication des partenaires. Une autre limite de l'étude provient du fait que l'évaluation du programme repose notamment sur une autoévaluation rapportée par les animatrices du programme. Ainsi, dans des recherches futures, il serait pertinent de trianguler ces données avec celles provenant d'un observateur externe, exempt de biais. En dépit de ces limites, la mise en œuvre de ce programme souligne la faisabilité des interventions de groupe en milieu scolaire primaire, une avenue de choix pour limiter le développement des problèmes liés à l'anxiété à l'école. Sur la base des recommandations qui découlent de cette première implantation, les milieux scolaires primaires pourront donc adapter celui-ci à leur convenance et selon leurs besoins, pour ainsi contribuer à limiter les problématiques liées aux comportements intériorisés.

\section{BIBLIOGRAPHIE}

Achenbach, T. M. et Rescorla, L. A. (2001). Manual for the ASEBA school-age forms \& profiles: An integrated system of multi-informant assessment. Burlington, VT: University of Vermont, Research Center for Children, Youth, \& Families.

Albano, A. M., Chorpita, B. F. et Barlow, D. H. (2003). Childhood anxiety disorders. Dans E. J. Mash et R. A. Barkley (éds.), Child psychopathology (pp. 279-329). New York, NY: Guilford Press.

Barlow, D. H., Allen, L. B. et Choate, M. L. (2004). Toward a unified treatment for emotional disorders. Behavior Therapy, 35(2), 205-230. https://doi.org/10.1016/s00057894(04)80036-4

Barlow, D. H., Farchione, T. J., Fairholme, C. P., Ellard, K. K., Boisseau, C. L., Allen, L. B. et Ehrenreich-May, J. (2011). Unified protocol for transdiagnostic treatment of emotional disorders: Therapist guide. New York, NY: Oxford University Press.

Barrett, P. et Ollendick, T. H. (2004). Handbook of interventions that work with children and adolescents: Prevention and treatment. Chichester: John Wiley et Sons Ltd.

Bluteau, J. (2017). Validation d’un programme de développement de compétences pour faire face au stress chez des adolescents présentant des troubles intériorisés. Thèse de doctorat. Université du Québec à Trois-Rivières.

Chen, H. (2005). Practical program evaluation: Assessing and improving planning, implementation and effectiveness. Thousand Oaks, CA: Sage Publications.

CISSS Centres jeunesse de Laval. (2016). Programme In vivo : évaluation de la mise en œuvre. Laval, Québec: CISSS Laval.

Dickerson, S. S. et Kemeny, M. E. (2004). Acute stressors and cortisol responses: A theoretical integration and synthesis of laboratory research. American Psychological Association, 130(3), 355-391. https://doi.org/10.1037/0033-2909.130.3.355. Medline:15122924

Dufour, M.-M. et Bluteau, J. (2016a). Évaluation de l'implantation du programme In vivo Institut Thérapeutique, Éducatif et Pédagogique d'Angoulême. Montréal, Québec: UQAM.

Dufour, M.-M. et Bluteau, J. (2016b). Évaluation de l'implantation du programme In vivo : CISSS-Centres jeunesse de Lanaudière. Montréal, Québec: UQAM. 
Dufour, M.-M. et Bluteau, J. (2016c). Évaluation de l'implantation du programme In vivo : CISSS-Centres jeunesse des Laurentides. Montréal, Québec: UQAM.

Dumas, J. E. (2012). L'enfant anxieux. Comprendre la peur de la peur et redonner courage. Bruxelles: De Boeck Éditions.

Fréchette-Simard, C., Plante, I. et Bluteau, J. (2018). Strategies included in cognitive and behavioral therapy programs to treat internalized disorders: A systematic review. Cognitive Behaviour Therapy, 47(4), 262-285. https://doi.org/10.1080/16506073.201 7.1388275. Medline:29103355

Gamache, V., Joly, J. et Dionne, C. (2011). La fidélité d'implantation du programme québécois d'intervention comportementale intensive destiné aux enfants ayant un trouble envahissant du développement en CRDITED. Revue de psychoéducation, 40(1), 1-23.

Girolamo, A. (2018). Une meilleure capacité à faire face au stress pour une réduction de l'agressivité réactive chez les élèves $d u$ secondaire : évaluation du programme In vivo. Mémoire de maîtrise. Université du Québec à Montréal.

Grills-Taquechel, A. E., Fletcher, J. M., Vaughn, S. R., Denton, C. A. et Taylor, P. (2013). Anxiety and inattention as predictors of achievement in early elementary school children. Anxiety Stress Coping, 26(4), 391-410. https://doi.org/10.1080/10615806.20 12.691969. Medline:22764776

Joly, J. (2008). Questionnaire d'autoévaluation de la fidélité d'implantation d'un programme. Communication personnelle, Mai, 2008.

Joly, J., Tourigny, M. et Thibaudeau, M. (2005). La fidélité d'implantation des programmes de prévention ou d'intervention dans les écoles auprès des élèves en difficulté de comportements. Nouveaux cahiers de la recherche en éducation, 8(2). 101-110. https://doi. org/10.7202/1017533ar

Katzman, M., Bleau, P., Blier, P., Chokka, P., Kjernisted, K.,Van Ameringen, M., ... l'Université McGill. (2014). Canadian clinical practice guidelines for the management of anxiety, posttraumatic stress and obsessive-compulsive disorder. BioMed Central Psychatric, 14(Suppl 1), 1-83. https://doi.org/10.1186/1471-244x-14-s1-s1. Medline:25081580

Kazdin, A. E., Siegel, T. C. et Bass, D. (1992). Cognitive problem-solving skills training and parent management training in the treatment of antisocial behavior in children. Journal of Consulting and Clinical Psychology, 60(5), 733-747. https://doi. org/10.1037//0022-006x.60.5.733. Medline:1401389

Lecompte, V., Moss, E., Cyr, C. et Pascuzzo, K. (2014). Preschool attachment, self-esteem and the development of preadolescent anxiety and depressive symptoms. Attachment and Human Development, 16(3), 242-260. https://doi.org/10.1080/14616734.2013.8 73816. Medline:24417266

Lupien, S. J., King, S., Meany, M. J. et McEwen, B. S. (2001). Can poverty get under your skin? Basal cortisol levels and cognitive function in children from low and high socioeconomic status. Development and Psychopathology, 13(3), 653-676. https://doi. org/10.1017/s0954579401003133. Medline:11523853

Mason, J. W. (1968). A review of psychoendocrine research on the pituitary-adrenal cortical system. Psychosomatic Medecin, 30(5), 576-607. https://doi.org/10.1097/00006842196809000-00020. Medline:4303377 
McLaughlin, K. A. et Hatzenbuehler, M. L. (2009). Stressful life events, anxiety sensitivity, and internalizing symptoms in adolescents. Journal of Abnormal Psychology, 118(3), 659-669. https://doi.org/10.1037/a0016499. Medline:19685962

Ministère du conseil exécutif du Gouvernement du Québec. (2013). Le programme In vivo : une approche innovante en santé mentale chez les jeunes. Bulletin prospective, 17(1). Récupéré le 9 décembre 2015 de. https://w3.mce.gouv.qc.ca/SPPS-Bulletin/ AfficherArticle.asp? $\mathrm{Cle}=80$

Narusyte, J., Ropponen, A., Alexanderson, K. et Svedberg, P. (2017). Internalizing and externalizing problems in childhood and adolescence as predictors of work incapacity in young adulthood. Social Psychiatry and Psychiatric Epidemiology, 52(9), 1159-1168. https://doi.org/10.1007/s00127-017-1409-6. Medline:28638965

Paquette, G., Joly, J. et Tourigny, M. (2009). La fidélité de l'implantation d’un programme d'intervention pour adolescentes agressées sexuellement : une mesure par observation systématique. The Canadian Journal of Program Evaluation, 24(2), 57-79.

Piché, G., Cournoyer, M., Bergeron, L., Clément, M. et Smolla, N. (2017). Épidémiologie des troubles dépressifs et anxieux chez les enfants et les adolescents québécois. Santé mentale au Québec, 42(1), 19-42. https://doi.org/10.7202/1040242ar

Weissman, A., Antinoro, D. et Chu, B. (2009). Cognitive-behavioral therapy for anxious youth in school settings. Advances and challenges. Dans M. Mayer, R. Van Acker, J. Lochman, et F. Gresham (éds.), Cognitive-behavioral interventions for emotional and behavioral disorders: School Based Practice (pp. 173-203). New York, NY: Guilford Press.

Wood, J. (2006). Effect of anxiety reduction on children's school performance and social adjustment. Developmental Psychology, 42(2), 345-349. https://doi.org/10.1037/00121649.42.2.345. Medline: 16569172

Zahn-Waxler, C., Klimes-Dougan, B. et Slattery, M. J. (2000). Internalizing problems of childhood and adolescence: Prospects, pitfalls, and progress in understanding the development of anxiety and depression. Development and Psychopathology, 12(3), 443-466. https://doi.org/10.1017/s0954579400003102. Medline:11014747

\section{PRÉSENTATION DES AUTEURS}

Catherine Fréchette-Simard complète actuellement un doctorat en éducation à l'Université du Québec à Montréal. Ses recherches portent sur les comportements intériorisés et l'anxiété de performance à lécole primaire et secondaire, de même que les liens entre ces problématiques et la motivation scolaire de l'élève. Elle est également coordonnatrice de la Chaire de recherche sur l'égalité des genres à l'école et œuvre à différents projets portant sur cette thématique.

Jonathan Bluteau est psychoéducateur et professeur agrégé au département deéducation et formation spécialisées à l'Université du Québec à Montréal (UQAM). Le professeur Bluteau détient un doctorat en psychoéducation et se spécialisé dans l'élaboration et l'évaluation de programmes d'intervention pour adolescents ayant des troubles anxio-dépressifs. Ses intérêts de recherche ciblent la prévention des problèmes de santé mentale chez les jeunes, létude des biomarqueurs du stress liés à la santé mentale, ainsi que le phénomène du surdiagnostic et du surtraitement médical en santé mentale. Sa posture, ses projets 
d'intervention et ses projets de recherche s'appuient sur les théories de lévolution et la biologie évolutive. Récemment, il s'intéresse aux impacts de l'architecture des écoles et l'aménagement de l'environnement de la classe sur le bien-être des élèves et des enseignants. Il travaille et intervient auprès de trois réseaux en France, soit l'Association française de thérapie cognitive et comportementale, le réseau des Instituts thérapeutiques éducatifs et pédagogiques, ainsi que l'Association française des psychologues de l'éducation nationale. Il est membre de l'Ordre des psychoéducateurs et psychoéducatrices du Québec, de l'Institut santé et société de l'UQAM, de la Société québécoise d'évaluation de programme (SQEP) et de la Société canadienne d'évaluation (SCE). Également, il est chercheur régulier au Centre d'études sur l'apprentissage et la performance UQAM (CEAP)

Isabelle Plante est professeure au département d'éducation et formation spécialisées de l'Université du Québec à Montréal et chercheure régulière au Centre d'études sur l'apprentissage et la performance (CEAP). Titulaire de la Chaire de recherche sur l'égalité des genres à lécole (CRÉGÉ), ses travaux se centrent sur la compréhension des facteurs qui caractérisent les parcours scolaires différentiés des garçons et des filles du primaire et du secondaire. Elle s'intéresse notamment au rôle des stéréotypes sociaux de genre à propos des capacités cognitives selon le sexe dans la motivation, la réussite et les aspirations professionnelles des garçons et des filles. Ses recherches traitent également des problématiques typiquement vécues selon le genre à l'école, comme l'anxiété de performance chez les filles et les troubles de comportement extériorisés chez les garçons. 